\title{
RECORD-SETTING COSMIC-RAY INTENSITIES IN 2009 AND 2010
}

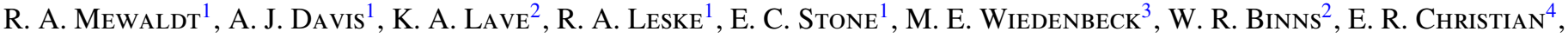 \\ A. C. Cummings ${ }^{1}$, G. A. de NOlfo $^{4}$, M. H. Israel ${ }^{2}$, A. W. Labrador ${ }^{1}$, And T. T. von Rosenvinge ${ }^{4}$ \\ ${ }^{1}$ California Institute of Technology, Pasadena, CA 91125, USA \\ ${ }^{2}$ Washington University, St. Louis, MO 63130, USA \\ 3 Jet Propulsion Laboratory, California Institute of Technology, Pasadena, CA 91109, USA \\ ${ }^{4}$ NASA/Goddard Space Flight Center, Greenbelt, MD 20771, USA \\ Received 2010 June 5; accepted 2010 September 13; published 2010 October 7
}

\begin{abstract}
We report measurements of record-setting intensities of cosmic-ray nuclei from $\mathrm{C}$ to $\mathrm{Fe}$, made with the Cosmic Ray Isotope Spectrometer carried on the Advanced Composition Explorer in orbit about the inner Sun-Earth Lagrangian point. In the energy interval from $\sim 70$ to $\sim 450 \mathrm{MeV}$ nucleon $^{-1}$, near the peak in the near-Earth cosmic-ray spectrum, the measured intensities of major species from C to Fe were each 20\%-26\% greater in late 2009 than in the 1997-1998 minimum and previous solar minima of the space age (1957-1997). The elevated intensities reported here and also at neutron monitor energies were undoubtedly due to several unusual aspects of the solar cycle 23/24 minimum, including record-low interplanetary magnetic field (IMF) intensities, an extended period of reduced IMF turbulence, reduced solar-wind dynamic pressure, and extremely low solar activity during an extended solar minimum. The estimated parallel diffusion coefficient for cosmic-ray transport based on measured solar-wind properties was 44\% greater in 2009 than in the 1997-1998 solar-minimum period. In addition, the weaker IMF should result in higher cosmic-ray drift velocities. Cosmic-ray intensity variations at $1 \mathrm{AU}$ are found to lag IMF variations by $2-3$ solar rotations, indicating that significant solar modulation occurs inside $\sim 20 \mathrm{AU}$, consistent with earlier galactic cosmic-ray radial-gradient measurements. In 2010, the intensities suddenly decreased to 1997 levels following increases in solar activity and in the inclination of the heliospheric current sheet. We describe the conditions that gave cosmic rays greater access to the inner solar system and discuss some of their implications.
\end{abstract}

Key words: cosmic rays - solar wind - Sun: activity - Sun: heliosphere

\section{INTRODUCTION}

In 2008 January, the first solar-cycle 24 sunspot was reported and it appeared that the fifth solar minimum of the space era might be drawing to a close. At that time galactic cosmic-ray (GCR) intensities measured by NASA's Advanced Composition Explorer (ACE) were comparable to those in 1997 and had apparently leveled off (Figure 1(a)). A projection based on 55 years of ground-based neutron-monitor data (using a 10.5 year solar cycle) suggested that new solar activity would begin in early 2008. However, solar-minimum conditions continued through early 2010 (Figures 1(b) and (c)), and the Sun entered an unfamiliar state characterized by much weaker polar and interplanetary magnetic fields (IMFs; Smith \& Balogh 2008; Lee et al. 2009; Figure 1(d)) and by greatly reduced solar-wind density and dynamic pressure (McComas et al. 2008; Figure 1(f)). In response to these and related conditions, cosmic rays had much greater access to $1 \mathrm{AU}$ in 2009 than during the previous four solar minima, triggering neutron monitors at record levels (Ahluwalia \& Ygbuhay 2010), and producing intensities at $200 \mathrm{MeV}$ nucleon $^{-1}$ (near the peaks in $1 \mathrm{AU}$ cosmicray spectra) that were more than $20 \%$ greater than previously observed during the space era (Figure 1(a)).

The inner heliosphere is, to a large extent, shielded from cosmic rays by the expanding solar wind and its magnetic field, and the near-Earth GCR intensity varies over the solar cycle as a result of processes collectively referred to as "solar modulation". As cosmic rays diffuse inward they scatter off irregularities in the diverging IMF and lose energy (Parker 1966; Gleeson \& Axford 1968). Cosmic rays also undergo gradient and curvature drifts in the large-scale IMF (Jokipii \& Thomas 1981; Ferreira $\&$ Potgieter 2004). During the current solar minimum, positive particles drift inward along the current sheet and, near the Sun, drift poleward. Approximately every 11 years the Sun's dipole magnetic field reverses direction, reversing the drift patterns. Since the last reversal in 2001, the Sun's field in the northern hemisphere has had predominantly negative (inward-pointing) polarity (denoted as an " $A<0$ " cycle).

$A C E$ was launched during the 1997 solar minimum and since early 1998 has orbited the inner-Lagrangian (L1) point carrying nine solar energetic particle (SEP), cosmic-ray, solar-wind, and magnetic-field instruments (Stone et al. 1998a). The Cosmic Ray Isotope Spectrometer (CRIS) measures GCR nuclei from He to $\mathrm{Zn}(2 \leqslant Z \leqslant 30)$ from $\sim 50$ to $\sim 500 \mathrm{MeV}$ nucleon ${ }^{-1}$ with unprecedented precision (Stone et al. 1998b). In contrast, the response of sea-level, high-latitude neutron monitors (Clem \& Dorman 2000) begins at $\sim 1 \mathrm{GV}(\sim 433 \mathrm{MeV}$ for protons) with a median response $\geqslant 10 \mathrm{GV}$ (Ahluwalia \& Fikani 2007). George et al. (2009) reported GCR measurements of B to Ni nuclei during the 1997-1998 solar minimum and subsequent solar maximum. This paper documents how the 2009-2010 GCR intensities of cosmic-ray nuclei from $\mathrm{H}$ to $\mathrm{Fe}$ exceed those in recent solar minima, and discusses conditions leading to this excess.

\section{OBSERVATIONS}

The CRIS instrument team measures cosmic-ray spectra for elements from $\mathrm{B}$ to $\mathrm{Ni}$ that stop in four detector stacks and reports these in seven energy intervals (see http://www.srl.caltech.edu/ACE/ASC/level2/index.html). This study uses intensities averaged over 27 day Bartels rotations, excluding days contaminated by SEPs (George et al. 2009). Heavy species like $\mathrm{Fe}(Z=26)$ that stop in the detector stacks 

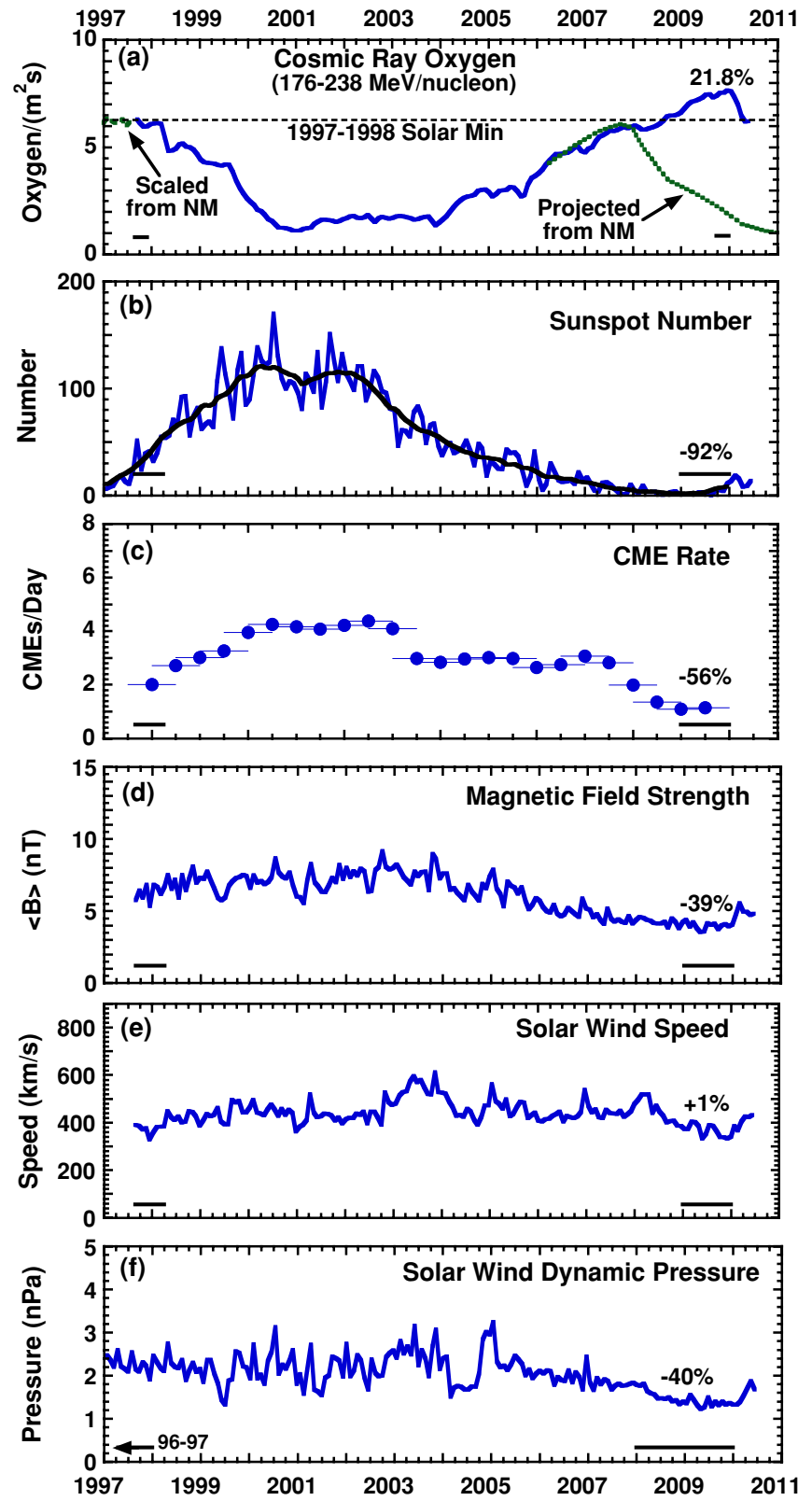

Figure 1. (a) Cosmic-ray oxygen intensity (rolling averages of two Bartels rotations) over the ACE mission. Also shown are 1996-1997 oxygen intensities scaled from Climax neutron-monitor data (http://ulysses.sr.unh.edu/ NeutronMonitor/neutron_mon.html) and a 2006-2012 oxygen rate projected using 1951 to 2005 Climax data and a 10.5 year solar cycle. The late 2009 intensity was $21.8 \%$ greater than the 1997-1998 maximum (dashed line). (b) Monthly and smoothed sunspot numbers from the Royal Observatory of Belgium (http://sidc.oma.be/); (c) CME rates from $\mathrm{SOHO} / \mathrm{LASCO}$ (http://cdaw.gsfc.nasa.gov/CME_list/); (d) IMF strength from ACE/MAG; (e) solar-wind speed from $A C E / S W E P A M$ and NASA's OmniWeb; and (f) the solar-wind dynamic pressure (NASA OmniWeb). The percentage change of each parameter from the last minimum to the present is indicated (based on the periods indicated by horizontal bars).

have higher average energy per nucleon than lighter species. However, $\sim 200 \mathrm{MeV}$ nucleon $^{-1}$ is within the stopping-range of all species reported here. Figure 2(a) shows time variations of six abundant "primary" species (most observed particles were accelerated from interstellar material). These species are relatively free of "secondaries" produced by nuclear interactions with interstellar gas and have similar energy spectra (George et al. 2009). All six species have similar intensity histories with a mean excess of $23.3 \% \pm 0.9 \%$ in late 2009 .
Although $A C E$ instruments do not measure cosmic-ray proton spectra at $\sim 200 \mathrm{MeV}$, the integral proton intensity can be monitored using the bottom detectors in the four solid-state detector stacks (labeled "E9" in Figure 11 of Stone et al. 1998b). These large-area devices $\left(68 \mathrm{~cm}^{2}\right.$ each) respond to charged particles (including minimum-ionizing species) from all incident directions. The estimated threshold for $\mathrm{H}$ and ${ }^{4} \mathrm{He}$ averages $\sim 120 \mathrm{MeV}$ nucleon $^{-1}$ over the instrument's upper hemisphere (the threshold for the spacecraft-facing side is similar but harder to assess). During 1997-2008 there is an excellent correlation between the E9 count rate and $>120 \mathrm{MeV}$ proton intensities from the BESS balloon-borne instrument (Shikaze et al. 2007) and PAMELA mission (Casolino et al. 2009), relating the E9 count rate and $>120 \mathrm{MeV}$ proton intensity with $\sim 2 \%$ accuracy.

Relative 27 day rates of $>120 \mathrm{MeV}$ protons and $200 \mathrm{MeV}$ nucleon $^{-1}$ oxygen are compared in Figure 2(b). The two rates track each other closely; the proton rate varies less because it is integrated to high energies. In late 2008, both rates began exceeding 1997-1998 levels. At the end of 2009 the inferred $>120 \mathrm{MeV}$ proton intensity was $13.7 \%$ greater than in 1997 September, but it decreased in early 2010 following changes in several interplanetary parameters.

To establish that 2009 cosmic-ray intensities $<1 \mathrm{GeV}$ nucleon $^{-1}$ are the highest of the space era we compare $\mathrm{He}$ intensities over five solar minima (Figure 3). Although no single spacecraft covered this period, there are IMP- 8 data from the Goddard Space Flight Center instrument from 1973 to 2001 and earlier data from several spacecraft. To extend the $I M P-8$ $\mathrm{He}$ intensities, we normalize to CRIS $\mathrm{C}$ using a $\mathrm{He} / \mathrm{C}$ ratio of 42.6 at $100-200 \mathrm{MeV}$ nucleon ${ }^{-1}$. The peak He intensities (3 rotation averages) in the 1997-1998 and 1976-1977 $(A>0)$ minima agree to within $2 \%$. The He estimates for late 2009 exceed those in 1997 by $20 \% \pm 2 \%$ and those in 1965 and 1987 by $\sim 40 \%$.

We note that solar-minimum measurements of cosmic-ray ionization in the upper atmosphere during the 1930s and 1940s were $\sim 10 \%-15 \%$ greater than for the 1965 and 1975 solar minima (Neher 1971; McCracken \& Beer 2007). However, there are no measured intensities or energy spectra for individual cosmic-ray species from this era for comparison with those from 2009-2010.

Energy spectra of C and Fe for 1997 and 2009 are shown in Figure 4, along with calculated spectra from a GCR transport model and a spherically symmetric solar-modulation code that includes diffusion, convection, and adiabatic deceleration (Fisk 1971; Appendix C.2 of George et al. 2009). Interstellar energy spectra from the transport code are input to the modulation model. Although we use Fisk's numerical solution and not the "force-field solution" (Gleeson \& Axford 1968), it is convenient to label each model spectrum by the modulation parameter introduced by Gleeson \& Axford ( $\phi$, in MV), representing the integral of $V_{\mathrm{SW}} /(3 \mathrm{~K})$ from $1 \mathrm{AU}$ to the modulation boundary. Here $V_{\text {SW }}$ is the solar wind velocity ( $\sim 400 \mathrm{~km} \mathrm{~s}^{-1}$ in the ecliptic) and $K$ is a radial diffusion coefficient assumed proportional to $\beta \mathrm{R}$, where $\beta$ is particle velocity in units of the speed of light and $R$ is rigidity.

As expected, the 2009 Fe spectrum has a greater increase in intensity at $150 \mathrm{MeV}$ nucleon $^{-1}(\sim 24 \%)$ than at $\sim 360 \mathrm{MeV}_{\text {nucleon }}{ }^{-1}(\sim 16 \%)$. The model spectrum for $\mathrm{Fe}$ peaks at somewhat lower energy than the measurements. In addition, $\mathrm{Mg}, \mathrm{Si}$, and $\mathrm{Fe}$ spectra are better fit with smaller $\phi$ values than are $\mathrm{C}$ and $\mathrm{O}$ (see also Wiedenbeck et al. 2009). Comparing 

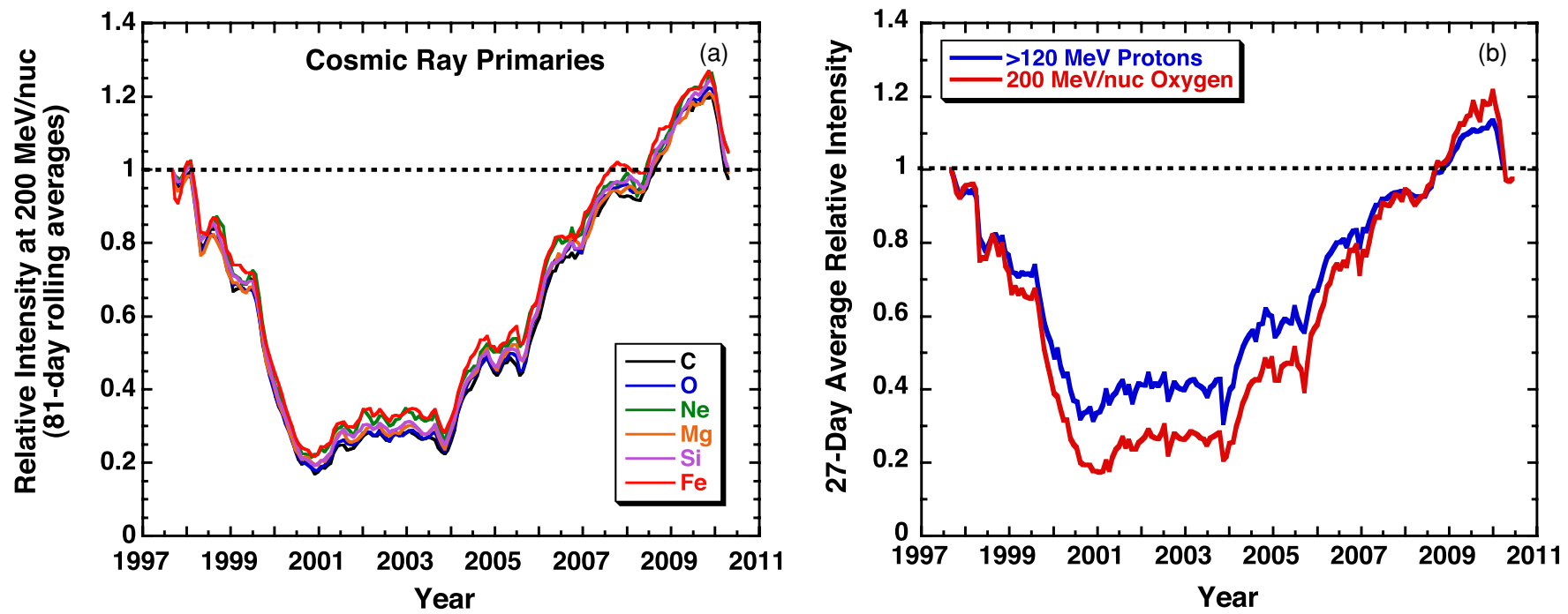

Figure 2. (a) Intensity of six abundant cosmic-ray species at $\sim 200 \mathrm{MeV}$ nucleon $^{-1}$ (rolling averages of three Bartels rotations) with each normalized to the first three rotations of the $A C E$ mission (rotations 2240-2242). (b) Twenty-seven day average intensities of $>120 \mathrm{MeV}$ protons (see the text) and $\sim 200 \mathrm{MeV}$ nucleon ${ }^{-1}$ oxygen, both normalized to unity during Bartels-rotation 2240 in 1997.

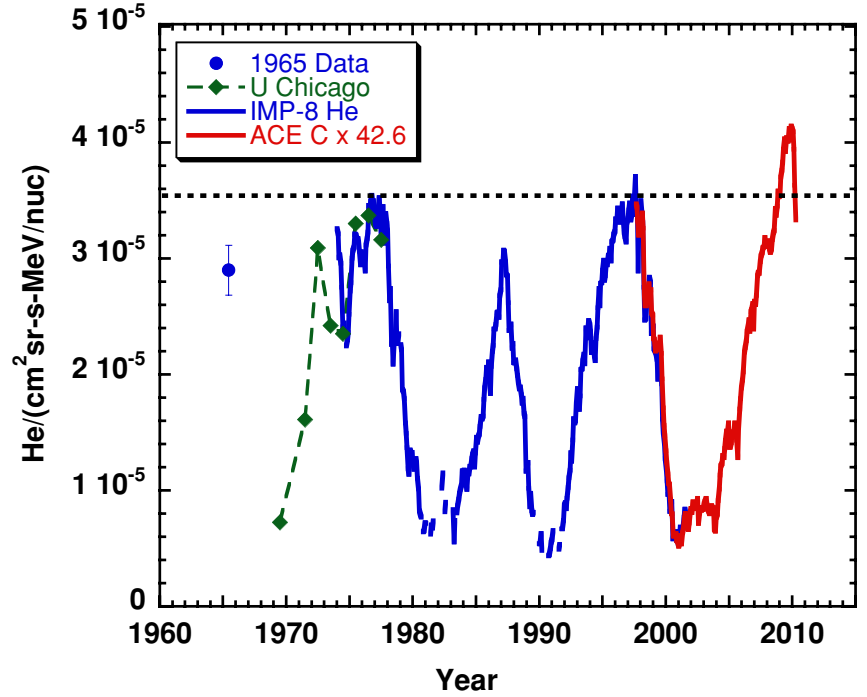

Figure 3. Intensity of $100-200 \mathrm{MeV}$ nucleon ${ }^{-1} \mathrm{He}$ from 1965 to 2010 . The 1965 point (blue circle) is based on a fit to several measurements (Figure 1(b) of Garcia-Munoz et al. 1983). University of Chicago data (green diamonds) are from Garcia-Munoz et al. (1975, 1977), Beatty et al. (1985), and Evenson et al. (1983). The 1974-2001 data (blue line) are from the IMP-8/GME instrument (R. McGuire 2001, private communication) and 1997-2010 data (red line) are scaled from $A C E / C R I S$ carbon measurements (see the text). The peak intensity in 2009 ( 81 day average) exceeded that in 1997 (dashed line) by 19.8\% $\pm 1.7 \%$.

modeled and measured intensities of the six primary species at $\sim 200 \mathrm{MeV}$ nucleon $^{-1}$, the average modulation levels $(\phi)$ are $\sim 320 \mathrm{MV}$ in late 1997 and $\sim 235 \mathrm{MV}$ in late 2009. While the spherically symmetric model gives a reasonable approximation to the data, the fits are not perfect, suggesting that a more realistic model including gradient and curvature drifts is ultimately required.

\section{CONDITIONS CONTRIBUTING TO THE 2009 INTENSITIES}

We now discuss several interplanetary parameters that collectively produced conditions for elevated intensities in 2009-2010.

\subsection{Interplanetary Magnetic Field Strength and Turbulence Level}

It is well known that cosmic-ray intensities are inversely correlated with the IMF strength, denoted by $B$ (Burlaga \& Ness 1998; Cane et al. 1999). Indeed, it is often assumed that the interplanetary cosmic-ray diffusion coefficient along the mean magnetic field $\left(K_{\|}\right)$is proportional to either $1 / B$ (Jokipii \& Davila 1981; Reinecke et al. 2000) or $1 / B$ to some power (Caballero-Lopez et al. 2004; Ferreira \& Potgieter 2004). Diffusion perpendicular to the magnetic field is often assumed to scale as $K_{\mathrm{II}}$ (Ferreira \& Potgieter 2004). In addition, GCR drift velocities increase with decreasing IMF strength (Jokipii \& Levy 1977; Jokipii \& Kota 1989). In 2009, the IMF averaged 3.9 nT compared with $6.3 \mathrm{nT}$ for 1997 September-1998 March (Figure 1(d)) and $~ 5.6 \mathrm{nT}$ during the 1987 solar minimum, implying that cosmic rays had greater access in 2009. The decrease in GCR intensity in early 2010 followed an IMF increase from $\sim 4$ to $\sim 5 \mathrm{nT}$ (Figures 1(a) and (d)).

Ahluwalia \& Ygbuhay (2010) found a good inverse correlation of 2006-2009 neutron-monitor data with monthly IMF averages. Figure 5(a) shows that the 27 day average oxygen intensities are also correlated with the IMF strength three solar rotations earlier (delays of $0,1,2$, and 4 rotations gave somewhat poorer correlations). The excellent correlation in Figure 5(a) indicates that solar-wind properties have considerable effect out to $\sim 20 \mathrm{AU}$, consistent with significant solar modulation occurring in the inner heliosphere at solar minimum (Fisk et al. 1998; Cummings \& Stone 1999; Fujii \& McDonald 2005).

Although Figure 5(a) shows that GCR intensities and the IMF are highly correlated, it does not reveal why. Cosmic-ray pitch-angle scattering in the IMF is regulated by turbulence in the IMF. Zank et al. (1998) find the mean free path (MFP) for diffusion along the mean magnetic field $\left(\lambda_{\|}\right)$is proportional to $B^{5 / 3} /\left(\delta B^{2}\right)$. Using the rms variation in the vector magnetic field for $\delta B$ (http://omniweb.gsfc.nasa.gov/ow.html) we find that $\lambda_{\|}$increased by $\sim 44 \%$ from $1997-1998$ to 2009 , and then suddenly decreased in early 2010, preceding a sudden drop in GCR intensity (Figure 5(b)). Diffusion perpendicular to the IMF may also have been more effective in 2009. 

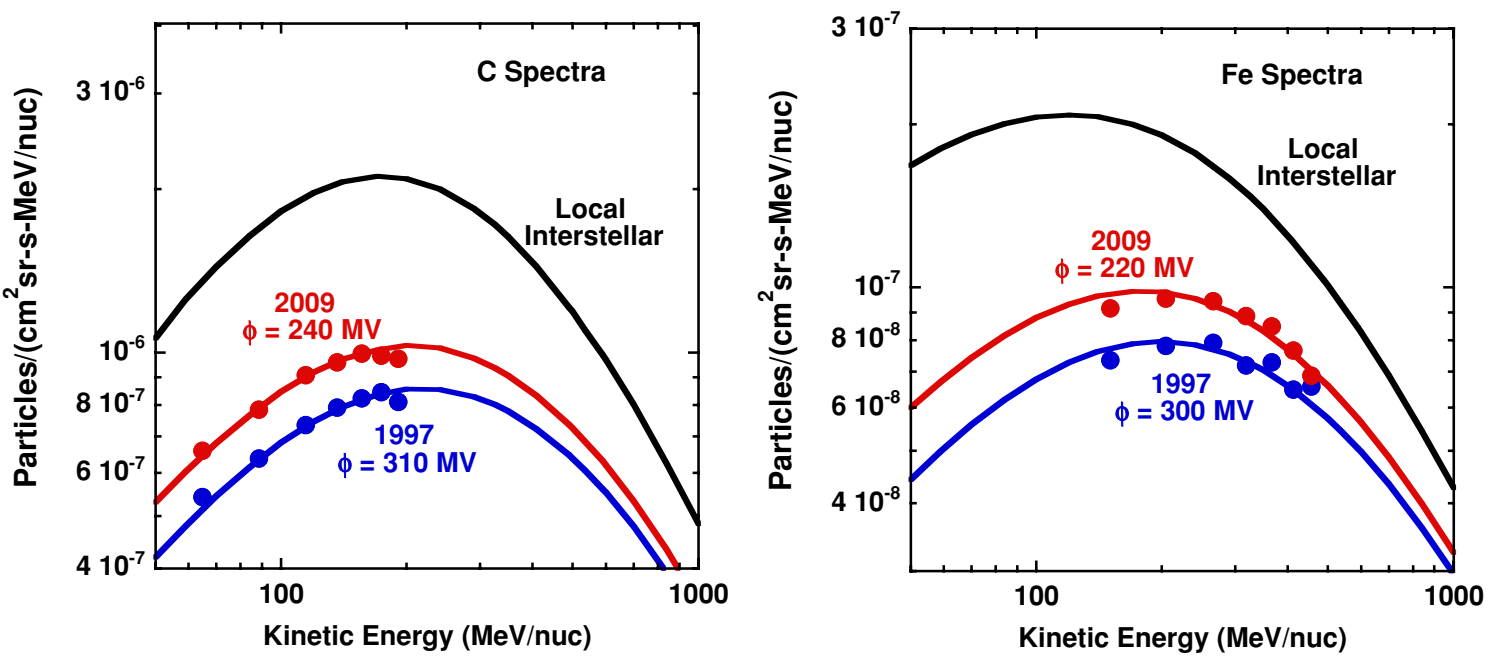

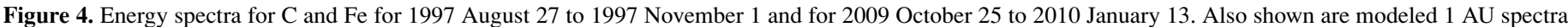

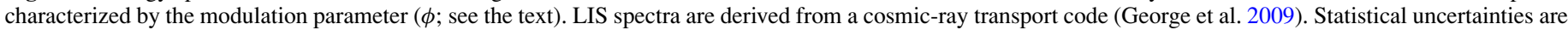
$\sim 1 \%-2 \%$ for $\mathrm{C}$ and $\sim 2 \%-3 \%$ for $\mathrm{Fe}$.
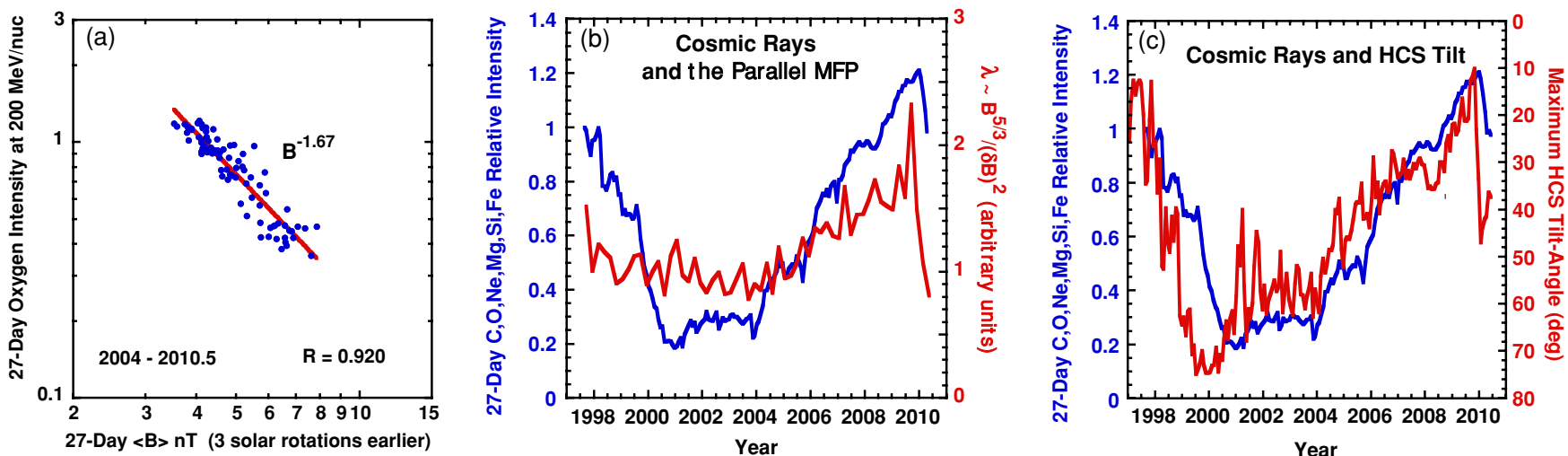

Figure 5. (a) Correlation of 27 day oxygen intensities from 2004-2010.5 with the 27 day IMF strength three solar rotations earlier. (b) Comparison of 81-day averages of the parallel mean free path (MFP; $\lambda \sim B^{5 / 3} /(\delta B)^{2}$; red line, see the text) with $\mathrm{C}+\mathrm{O}+\mathrm{Ne}+\mathrm{Mg}+\mathrm{Si}+\mathrm{Fe}$ intensities (each normalized to $1 / 6$ during Bartels rotation 2240 ; blue line); (c) the 27-day-average intensity of $\mathrm{C}+\mathrm{O}+\mathrm{Ne}+\mathrm{Mg}+\mathrm{Si}+\mathrm{Fe}$ (blue line) is plotted with the 27 -day average HCS tilt angle (red line; note inverse right-hand scale).

\subsection{Tilt of the Heliospheric Current Sheet}

In each of the previous three solar minima the heliospheric current sheet (HCS) tilt angle evolved from $>70^{\circ}$ at solar maximum to $<10^{\circ}$ at solar minimum (using the "classic" model at http://wso.stanford.edu/). During $A<0$ cycles, GCR ions enter the inner heliosphere largely by drifting along the HCS. When the HCS tilt angle (Figure 5(c)) is greater, GCR ions must drift a greater distance, and with a model that neglected diffusion, Jokipii \& Thomas (1981) calculated that the 1 AU GCR intensity would be inversely correlated with tilt angle (see also Potgieter et al. 2001; Ferreira \& Potgieter 2004). In the present solar minimum the tilt angle remained $>30^{\circ}$ until mid2009 when it finally decreased, eventually reaching $\sim 10^{\circ}$ to $\sim 13^{\circ}$ for three solar rotations.

The dip in GCR intensity in late 2007, followed by an upward surge in early 2008, appears related to HCS tilt angle changes (Figure 5(c)). The tilt angle decrease to $\sim 10^{\circ}$ in late 2009 was apparently less effective (see also Cummings \& Stone 1999), but it remained $<13^{\circ}$ for only $\sim 3$ rotations. The abrupt GCR decline in early 2010 is likely due in part to the tilt angle suddenly increasing from $\sim 10^{\circ}$ to $>40^{\circ}$ two or three rotations earlier.

In late 2008 , the Ulysses / KET $2.5 \mathrm{GV}$ proton rate $(\sim 1.7 \mathrm{GeV})$ was at 1997 solar-minimum levels with $\mathrm{a} \sim 35^{\circ}$ tilt angle. Heber et al. (2009) predicted a 30\% increase to record levels if the tilt angle reached $<10^{\circ}$. Record intensities did occur, although the increase from late 2008 to late 2009 at somewhat lower rigidities (1-2 GV) was $\sim 20 \%$ rather than $\sim 30 \%$ (Figures 2 and $5(\mathrm{c})$ ).

\subsection{Coronal Mass Ejection Rate}

Interplanetary coronal mass ejections (ICMEs) typically include stronger magnetic fields than ambient solar wind and several ICMEs can coalesce to inhibit GCR entry into the inner heliosphere (Burlaga \& Ness 1998). The 2008-2009 CME rates were noticeably lower than in 1997-1998 (Figure 1(c)). In addition, the average CME mass in 2008-2009 was $~ 10$ times smaller than in 1997-1998 (Vourlidas et al. 2010), thereby minimizing this solar-modulation contribution.

\subsection{Solar Wind Speed}

The solar-wind speed $\left(V_{\mathrm{SW}}\right)$ determines the rate cosmic rays are convected into the outer heliosphere and the adiabatic energy-loss rate in the solar wind. However, the average $V_{S W}$ in 2009 ( 375 $\mathrm{km} \mathrm{s}^{-1}$ ) was not unusual for solar minimum (late 1997 through early 1998 averaged $\sim 373 \mathrm{~km} \mathrm{~s}^{-1}$; Figure 1(e)). Given this small change in $V_{\mathrm{sw}}$ the effects on convection and energy loss should be minimal. 


\subsection{Solar Wind Dynamic Pressure}

McComas et al. (2008) reported that the solar wind dynamic pressure in 2008 was $\sim 22 \%$ lower than 11 years earlier, due to low-proton densities. In 2008-2009 the dynamic pressure continued to decrease (Figure 1(f)), becoming $\sim 40 \%$ lower in 2008-2009 than in 1996-1997. The balance between solarwind and pickup-ion dynamic pressure and interstellar-medium pressure regulates the termination shock and heliopause locations; with decreasing interplanetary pressure these boundaries contract. Although the solar wind takes $\sim 1$ year to reach the termination shock, it requires another year or more to reach the heliopause, often assumed to represent the GCR modulation boundary (e.g., Webber \& Higbie 2009). However, solarminimum intensity gradients for $<0.5 \mathrm{GeV}$ nucleon $^{-1} \mathrm{GCR}$ ions in the outer heliosphere are small compared to inside $~ 20 \mathrm{AU}$ (e.g., Figure 2 in Fisk et al. 1998), suggesting that boundarydistance changes have a minor effect on 1 AU GCR intensities.

\subsection{An Extended Solar Minimum}

Finally, the elevated 2009 GCR intensities are likely due in part to the extended solar minimum, which provided more time for GCRs to equilibrate than during recent $A<0$ minima. In contrast, during the 1987 minimum several neutron monitors only briefly reached their maximum count rates (Moraal et al. 1989). However, solar activity then increased and GCRs $<1 \mathrm{GeV}$ nucleon ${ }^{-1}$ may not have had an opportunity to equilibrate (Figure 3).

\section{DISCUSSION}

In some ways, the present minimum is reminiscent of 1987 (also $A<0$ ), when selected neutron monitors also reached their then all-time maximum intensities. However, 1987 was a weak minimum for GCRs $<1 \mathrm{GeV}$ nucleon $^{-1}$ (Figure 3) possibly because the IMF was $\sim 40 \%$ stronger than in 2009 or because the cosmic-ray diffusion coefficient had a different rigidity dependence. Our results, in combination with neutron-monitor data, show that in 2009 cosmic rays had greater access to $1 \mathrm{AU}$ over the full energy range from $\sim 0.1$ to $>10 \mathrm{GeV}^{\text {nucleon }}{ }^{-1}$.

Galactic cosmic rays affect both humans and hardware in space. We find 2009 GCR intensities at several hundred $\mathrm{MeV}$ nucleon $^{-1}$ more than $20 \%$ higher than previous direct measurements. Intensity increases at higher energies are expected to be smaller. By integrating modeled spectra that fit our observations we estimate that a $21.8 \%$ increase at $200 \mathrm{MeV}^{\text {nucleon }}{ }^{-1}$ (Figure 1(a)) corresponds to a $\sim 11 \%$ increase in the $>100 \mathrm{MeV}$ nucleon ${ }^{-1}$ GCR intensity of $A / Z \approx$ 2 nuclei. Hydrogen $(A / Z=1)$ varies somewhat more than $\mathrm{He}$ and we deduce an increase of $\sim 13.7 \%$ for $>120 \mathrm{MeV}$ protons. Integrating representative proton spectra times the energy-loss rate in $\mathrm{Si}$ indicates that the interplanetary radiation dose due to GCR protons increased by $14 \% \pm 2 \%$ from late 1997 to late 2009, an increase that appears consistent with 1997-2009 dose estimates in Schwadron et al. (2010), based on ACE/CRIS and neutron-monitor data.

Although the 2009 intensities are at a 50 year high level, measurements of ${ }^{10} \mathrm{Be}$ deposited in polar ice cores over the last $\sim 500$ years (McCracken et al. 2004a) indicate that the space era has occurred during a period of very low GCR intensity. Between the years $\sim 1400$ and $\sim 1900{ }^{10}$ Be production was typically $\sim 40 \%$ to $\sim 80 \%$ greater than in the early 1970 s. Measurements of ${ }^{10} \mathrm{Be}$ and ${ }^{14} \mathrm{C}$ also indicate higher-than-present
GCR intensities over most of the last $\sim 10,000$ years (Steinhilber et al. 2010). In addition, measurements of cosmic-ray ionization in the atmosphere (McCracken \& Beer 2007; Neher 1971) indicate that neutron-monitor rates in the 1930s (and perhaps the 1940s) would have exceeded those in recent minima, including 2009-2010.

Webber \& Higbie (2010) reported that Voyager $\mathrm{H}$ and He spectra in the heliosheath are increasing less fast than expected for some suggested interstellar spectra (e.g., Webber \& Lockwood 2001; Webber \& Higbie 2003; McCracken et al. 2004b). Rather, Webber \& Higbie $(2009,2010)$ favor interstellar spectra that roll over below $\sim 200 \mathrm{MeV}$ nucleon $^{-1}$ (Figure 4) as expected from ionization energy-loss effects (George et al. 2009). It is often assumed that if the Voyagers reach the heliopause they will sample local-interstellar (LIS) cosmicray spectra (e.g., Webber \& Higbie 2010). Knowledge of these spectra should provide benchmarks for interpreting long-term ${ }^{10} \mathrm{Be}$ and ${ }^{14} \mathrm{C}$ records by reducing uncertainties associated with LIS spectra and providing an upper limit to near-Earth cosmicray intensities in the past. In addition, knowledge of LIS spectra will provide improved estimates of the radiation environments that could occur during future human exploration beyond our magnetosphere.

\section{SUMMARY}

The observations reported here show that during the extended 2008-2010 solar minimum there was a 1.5 year period (2008 October through $2010 \mathrm{March}$ ) during which the intensity of heavy (He to Fe) GCRs with $\sim 70$ to $\sim 450 \mathrm{MeV}$ nucleon $^{-1}$ exceeded intensities during previous solar minima back to 1965, and most neutron monitors were also at record levels (Ahluwalia \& Ygbuhay 2010). The 2008-2010 solar minimum was unusual in several ways, including a greatly reduced IMF strength averaging 39\% less in 2009 than during 1997 September to 1998 March (Figure 1(d)) and a prolonged decrease in the interplanetary turbulence level. This combination produced a sustained increase in the estimated cosmic-ray MFP (Figure 5(b)) and also increased GCR drift velocities, allowing cosmic rays to penetrate more easily into the inner heliosphere than during other recent minima. The extended cycle $23 / 24$ solar minimum provides an excellent opportunity for state-of-the-art solar-modulation models to evaluate the relative effects of evolving solar/interplanetary properties. The long-term cosmic-ray record based on ${ }^{10} \mathrm{Be}$ and ${ }^{14} \mathrm{C}$ shows that the solar-cycle average GCR intensity at $1 \mathrm{AU}$ has been unusually low during the space era. It is possible that the near-Earth radiation environment is returning to more "normal" conditions.

This work was supported by NASA at Caltech (under grants NNX08AI11G and NNX10AE45G), the Jet Propulsion Laboratory, the Goddard Space Flight Center, and Washington University in St. Louis. We thank Wilcox Solar Observatory for making HCS data available, Robert McGuire for providing IMP-8/ GME data, and NASA's OmniWeb for providing solar wind data. We also appreciate the availability of neutron monitor data from the University of New Hampshire (under NSF grant ATM0339257), sunspot data from the Royal Observatory of Belgium, and CME data from the $\mathrm{SOHO} / \mathrm{LASCO} \mathrm{CME}$ Catalog. Finally, we thank the MAG, SWEPAM, and SWICS teams for providing data through the $A C E$ Science Center. 


\section{REFERENCES}

Ahluwalia, H. S., \& Fikani, M. M. 2007, J. Geophys. Res., 112, A08105

Ahluwalia, H. S., \& Ygbuhay, R. C. 2010, in AIP Conf. Proc. 1216, Twelfth International Solar Wind Conference, ed. M. Maksimovic et al. (Melville, NY: AIP), 699

Beatty, J. J., Garcia-Munoz, M., \& Simpson, J. A. 1985, ApJ, 294, 455

Burlaga, L. F., \& Ness, N. F. 1998, J. Geophys. Res., 103, 29719

Caballero-Lopez, R. A., Moraal, H., McCracken, K. G., \& McDonald, F. B. 2004, J. Geophys. Res., 109, A12102

Cane, H. V., Wibberenz, G., Richardson, I. G., \& von Rosenvinge, T. T. 1999, Geophys. Res. Lett., 26, 565

Casolino, M., et al. 2009, Nucl. Phys. B, 190, 293

Clem, J. M., \& Dorman, L. I. 2000, Space Sci. Rev., 93, 335

Cummings, A. C., \& Stone, E. C. 1999, Adv. Space Res., 23, 509

Evenson, P., Garcia-Munoz, M., Meyer, P., Pyle, K. R., \& Simpson, J. A. 1983, ApJ, 275, L15

Ferreira, S. E. S., \& Potgieter, M. S. 2004, ApJ, 603, 744

Fisk, L. A. 1971, J. Geophys. Res., 76, 221

Fisk, L. A., et al. 1998, Space Sci. Rev., 83, 179

Fujii, Z, \& McDonald, F. B. 2005, Adv. Space Res., 35, 611

Garcia-Munoz, M., Mason, G. M., \& Simpson, J. A. 1975, ApJ, 202, 265

Garcia-Munoz, M., Mason, G. M., \& Simpson, J. A. 1977, ApJ, 213, 263

Garcia-Munoz, M., Pyle, K. R., \& Simpson, J. A. 1983, ApJ, 274, L93

George, J. S., et al. 2009, ApJ, 698, 1666

Gleeson, L. J., \& Axford, W. I. 1968, ApJ, 154, 1011

Heber, B., et al. 2009, ApJ, 699, 1956

Jokipii, J. R., \& Davila, J. M. 1981, ApJ, 248, 1156

Jokipii, J. R., \& Thomas, B. 1981, ApJ, 243, 1115

Jokipii, J. R., \& Kota, J. 1989, Geophys. Res. Lett., 16, 1

Jokipii, J. R., \& Levy, E. H. 1977, ApJ, 213, L85

Lee, C. O., et al. 2009, Solar Phys., 256, 345
McComas, D. J., Ebert, R. W., Elliott, H. A., Goldstein, B. E., Gosling, J. T., Schwadron, N. A., \& Skoug, R. M. 2008, Geophys. Res. Lett., 35, L18103

McCracken, K. G., \& Beer, J. 2007, J. Geophys. Res., 112, A10101

McCracken, K. G., Beer, J., \& McDonald, F. B. 2004a, Adv. Space Res., 34 397

McCracken, K. G., McDonald, F. B., Beer, J., Raisbeck, G., \& Yiou, F. 2004b, J. Geophys. Res., 109, A12103

Moraal, H., Potgieter, M. S., \& Stoker, P. H. 1989, J. Geophys. Res., 94, 1459

Neher, H. V. 1971, J. Geophys. Res., 76, 1637

Parker, E. N. 1966, Planet. Space Sci., 14, 371

Potgieter, M. S., Burger, R. A., \& Ferreira, S. E. S. 2001, Space Sci. Rev., 97, 295

Reinecke, J. P. L., Moraal, H., \& McDonald, F. B. 2000, J. Geophys. Res., 105, 12651

Schwadron, N. A., Boyd, A. J., Kozarev, K., Golightly, M., Spence, H., Townsend, L. W., \& Owens, M. 2010, Space Weather, 8, S00E04

Shikaze, Y., et al. 2007, Astropart. Phys., 28, 154

Smith, E. J., \& Balogh, A. 2008, Geophys. Res. Lett., 35, L22103

Steinhilber, F., Abreu, J. A., Beer, J., \& McCracken, K. G. 2010, J. Geophys. Res., 115, A01104

Stone, E. C., Frandsen, A. M., Mewaldt, R. A., Christian, E. R., Margolies, D., Ormes, J. F., \& Snow, F. 1998a, Space Sci. Rev., 86, 1

Stone, E. C., et al. 1998b, Space Sci. Rev., 86, 285

Vourlidas, A., Howard, R. A., Patsourakos, S., Esfandiari, E., Yashiro, S., \& Michalek, G. 2010, ApJ, submitted (arXiv:1008.3737)

Webber, W. R., \& Higbie, P. R. 2003, J. Geophys. Res., 108, 1355

Webber, W. R., \& Higbie, P. R. 2009, J. Geophys. Res., 114, A02103

Webber, W. R., \& Higbie, P. R. 2010, J. Geophys. Res., 115, A05102

Webber, W. R., \& Lockwood, J. A. 2001, J. Geophys. Res., 106, 29323

Wiedenbeck, M. E., et al. 2009, Proc. 31st ICRC (Lodz), Paper 545

Zank, G. P., Matthaeus, W. H., Bieber, J. W., \& Moraal, H. 1998, J. Geophys. Res., 103, 2085 